\title{
Global minimizers for the Ginzburg-Landau functional below the first critical magnetic field
}

by

\author{
Etienne SANDIER ${ }^{\mathrm{a}, 1}$, Sylvia SERFATY ${ }^{\mathrm{b}, \mathrm{c}, 2}$ \\ ${ }^{a}$ Université François Rabelais, Département de Mathématiques, Parc Grandmont, \\ 37200 Tours, France \\ b CMLA, Ecole Normale Supérieure de Cachan, 61 avenue du Président Wilson, \\ 94235 Cachan Cedex, France \\ c Laboratoire d'Analyse Numérique et EDP, Bâtiment 425, Université de Paris-Sud, \\ 91405 Orsay, France
}

Manuscript received 5 October 1998

ABSTRACT. - We prove that the global minimizer of the GinzburgLandau functional of superconductors in an external magnetic field is, below the first critical field, the vortex-less solution found in (S. Serfaty, to appear).

(C) 2000 L'Association Publications de l'Institut Henri Poincaré. Published by Elsevier B.V. All rights reserved

RÉSUMÉ. - On montre que le minimiseur global de la fonctionelle de Ginzburg-Landau en supraconductivité, avec champ magnétique extérieur inférieur au premier champ critique est la solution sans vortex trouvée dans ( $\mathrm{S}$. Serfaty, à paraitre).

(C) 2000 L'Association Publications de l'Institut Henri Poincaré. Published by Elsevier B.V. All rights reserved

\footnotetext{
${ }^{1}$ E-mail: sandier@univ-tours.fr.

2 E-mail: serfaty@cmla.ens-cachan.fr.
} 


\section{INTRODUCTION}

We are interested in this paper in describing the minimizers of the Ginzburg-Landau functional

$$
J(u, A)=\frac{1}{2} \int_{\Omega}\left|\nabla_{A} u\right|^{2}+\left|h-h_{e x}\right|^{2}-h_{e x}^{2}+\frac{\kappa^{2}}{2}\left(1-|u|^{2}\right)^{2},
$$

that corresponds to the free energy of a superconductor in a prescribed, constant magnetic field $h_{e x}$. Here, $\Omega \subset \mathbb{R}^{2}$ is the smooth, bounded, and simply connected section of the superconductor; and the unknowns are the complex-valued order parameter $u \in H^{1}(\Omega, \mathbb{C})$ and the $U(1)$ connection $A \in H^{1}\left(\Omega, \mathbb{R}^{2}\right)$. The induced magnetic field $h$ is defined by $h=\operatorname{curl} A$. The order parameter $u$ indicates the local state of the material: $|u|$ is the density of superconducting electron pairs, so that, where $|u| \simeq 1$, the material is in its superconducting state, whereas where $|u| \simeq 0$, it is in its normal state. Finally $\kappa=1 / \varepsilon>0$ is the GinzburgLandau parameter depending on the material, and we are interested in the case of superconductors with high kappa. We stress that no boundary conditions are imposed on $(u, A)$, the characteristics of the solutions to the minimization problem are governed solely by $h_{e x}$.

Minimizers of $J(u, A)$ solve the associated Euler equations,

$$
\left\{\begin{aligned}
-\nabla_{A}^{2} u & =\kappa^{2} u\left(1-|u|^{2}\right) \\
-* d h & =\left(i u, d_{A} u\right)
\end{aligned}\right.
$$

It turns out that a key physical feature of solutions to (G.L.) is the existence, for a certain range of values of $h_{e x}$, of vortices, i.e., isolated zeros of $u$ at which $u$ has a nonzero degree: $u /|u|$ restricted to a small circle around the zero has a non-zero winding number as a map to the unit circle. Away from these zeros, $|u| \approx 1$. Describing solutions to (G.L.) then typically consists in describing the vortex structure of the solutions, i.e., to determine the number, degree and position of vortices.

The difficulty here is that without boundary conditions, there is no a-priori bound on the number of vortices. Even if boundary conditions are imposed, the problem of defining mathematically and describing the vortex structure of solutions is not an easy one. This was done in [3] for 
the functional

$$
F(u)=\frac{1}{2} \int_{\Omega}|\nabla u|^{2}+\frac{1}{2 \varepsilon^{2}}\left(1-|u|^{2}\right)^{2}
$$

supplemented by a Dirichlet boundary condition $g: \partial \Omega \rightarrow S^{1}$. There, a vortex structure is shown to exist for minimizers (and even critical points) of $F(u)$ when $\varepsilon \rightarrow 0$. Moreover it is proved that there are exactly $d=$ $|\operatorname{deg}(g)|$ vortices, and their position is determined. In [5] the functional (1.1) is studied with $h_{e x}$ set to zero and replaced by a gauge invariant variant of the Dirichlet condition. There again the vortex structure is shown to exist when $\varepsilon$ is small, and results similar to those in [3] are obtained about the number and position of the vortices.

In $[13,14]$ and [15], the second author studied minimizers of (1.1) without boundary condition. For more details on the notations and physical description of superconductors, we refer to [13] and the references therein. Let us just say that it is observed that for small values of the applied field $h_{e x}$, the material is superconducting everywhere (there are no vortices), the magnetic field does not penetrate it and approximately satisfies the London equation

$$
\left\{\begin{aligned}
-\Delta h+h & =0 & & \text { in } \Omega, \\
h & =h_{e x} & & \text { on } \partial \Omega .
\end{aligned}\right.
$$

This state is called the Meissner state, corresponding to vortex-less solutions in the terminology of [13] and [14], the corresponding solution $(u, A)$ to (G.L.) being called the Meissner solution. For $h_{e x}$ higher than some critical value $H_{c_{1}}$, the vortex-less solution is no longer energy minimizing.

In [13], to replace the absence of boundary conditions and thus the lack of a-priori estimates on the number of vortices, the functional $J(u, A)$ was minimized on a subdomain of $H^{1}(\Omega, \mathbb{C}) \times H^{1}\left(\Omega, \mathbb{R}^{2}\right)$. More precisely, choosing some (large) number $\mathcal{M}>0$, the minimization was performed on

$$
D=\left\{\left.(u, A)\left|\frac{1}{2} \int_{\Omega}\right| \nabla u\right|^{2}+\frac{1}{2 \varepsilon^{2}}\left(1-|u|^{2}\right)^{2}<\mathcal{M}|\log \varepsilon|\right\},
$$

and the following theorem was proved: 
THEOREM 1 [13]. - There exist $k_{1}=1 /\left(2 \max \left|\xi_{0}\right|\right), k_{2}^{\varepsilon}=\mathrm{O}_{\varepsilon}(1)$ and $k_{3}^{\varepsilon}=\mathrm{o}_{\varepsilon}(1)$, such that

$$
H_{c_{1}}=k_{1}|\log \varepsilon|+k_{2}^{\varepsilon},
$$

and $\varepsilon_{0}(\mathcal{M})$ such that for $\varepsilon<\varepsilon_{0}$, the following holds:

- if $h_{e x} \leqslant H_{c_{1}}$, a solution of (G.L.) that is minimizing in D exists, and satisfies $\frac{1}{2} \leqslant|u| \leqslant 1$;

- if $H_{c_{1}}+k_{3}^{\varepsilon} \leqslant h_{e x} \leqslant H_{c_{1}}+\mathrm{O}_{\varepsilon}(1)$, a solution of (G.L.) that is minimizing in $D$ exists, it has a bounded positive number of vortices $a_{i}^{\varepsilon}$ of degree one, such that $\operatorname{dist}\left(a_{i}^{\varepsilon}, \Lambda\right) \rightarrow 0$ where

$$
\Lambda=\left\{x \in \Omega|| \xi_{0}(x)|=\max | \xi_{0} \mid\right\},
$$

and $\exists C>0, \operatorname{dist}\left(a_{i}^{\varepsilon}, a_{j}^{\varepsilon}\right) \geqslant C$ for $i \neq j$, i.e., the $a_{i}$ 's tend to distinct points $\in \Lambda$.

In addition, it is proved in [15] that the Meissner solution found for $h_{e x} \leqslant H_{c_{1}}$ still exists for $h_{e x} \geqslant H_{c_{1}}$ (even though it is then only locally minimizing in $D$ ), and is unique among vortex-less solutions.

$H_{c_{1}}$ is known as the first critical field. It is the value of $h_{e x}$ for which the energy of the Meissner solution becomes equal to the energy of a single-vortex configuration. Here, we wish to know whether or not, for $h_{e x} \leqslant H_{c_{1}}$, the Meissner solution is a global minimizer of the energy in addition to being a minimizer in $D$.

This question arises naturally for all the vortex solutions found in [13] and [14] that are minimizers in $D$, but that are all likely to be global minimizers. However, the proof in [13] and [14] uses repeatedly the apriori bound on the number of vortices given by (1.4). Here, without this upper bound, we are still able to prove a result about vortex-less solutions:

THEOREM 1. - There exists a value $H_{c_{1}}^{\prime} \simeq H_{c_{1}}$ (more precisely $H_{c_{1}}^{\prime}=$ $\left.H_{c_{1}}+\mathrm{O}(|\log | \log \varepsilon||)\right)$, such that, for sufficiently small $\varepsilon$, if $h_{e x} \leqslant H_{c_{1}}^{\prime}$, a globally minimizing solution of (G.L.) satisfies $|u| \geqslant 3 / 4$ on $\Omega$, and coincides with the solution found in Theorem 1 of [13].

Thus, we answer positively the question, though we have an imprecision on $H_{c_{1}}$ that we were not able to avoid.

In order to prove this theorem, we consider a minimizing solution of (G.L.) and assume it has possible vortices. We use a technique of R. Jerrard [7] to construct balls $B_{i}=B\left(a_{i}, r\right)$ of size $r \simeq 1 /|\log \varepsilon|^{\alpha}$ with sufficiently high $\alpha$, such that 


$$
\begin{aligned}
& |u| \geqslant \frac{3}{4} \quad \text { on } \Omega \backslash \bigcup_{i} B_{i}, \\
& d_{i}=\operatorname{deg}\left(u, \partial B_{i}\right), \\
& F_{\varepsilon}\left(u, B_{i}\right)>\pi\left|d_{i}\right| \log \frac{r}{\varepsilon},
\end{aligned}
$$

where we use the notation

$$
F_{\varepsilon}(u, V)=\frac{1}{2} \int_{V}|\nabla u|^{2}+\frac{1}{2 \varepsilon^{2}}\left(1-|u|^{2}\right)^{2} .
$$

The lower bound (1.7) was known to be true, see [3], under some assumptions on the restriction of $u$ on $\partial B_{i}$. Here, adapting slightly the techniques in [7], we are able to avoid making these assumptions and to construct these balls even though, in contrast to [13,14] and [15], their number is not bounded a priori independently of $\varepsilon$.

Then the key argument of the proof is to split conveniently the energy in a way that is similar to, but slightly different from the one used in [13], in order to obtain a lower bound of the energy on $\Omega \backslash \bigcup_{i} B_{i}$. Precisely, we find that for a minimizer $(u, A)$,

$$
J(u, A) \geqslant J_{0}+2 \pi h_{e x} \sum_{i} d_{i} \xi_{0}\left(a_{i}\right)+\sum_{i} F_{\varepsilon}\left(u, B_{i}\right)+\mathrm{o}(1),
$$

as $\varepsilon \rightarrow 0$. In the above expansion $J_{0}$ is roughly the minimal energy of a vortex-less configuration; and $\xi_{0}$ is a negative function depending only on the domain $\Omega$ (see [13] or Section 2). Putting together (1.7) and (1.8), and using the fact that the energy of a minimizer must be no greater than $J_{0}$ allows to conclude that vortices are not present if $h_{e x}$ is less than some number

$$
H_{c_{1}}^{\prime} \approx \frac{1}{2 \max \left|\xi_{0}\right|}|\log \varepsilon|,
$$

the right-hand side is precisely the $H_{c_{1}}$ computed in [13].

The idea is the same as in [13]: a vortex of degree $d$ "costs" almost $\pi|d||\log \varepsilon|$ to make (see (1.7)), while it can decrease $J(u, A)$ by at most $\pi d h_{e x} \xi_{0}\left(a_{i}\right)$ (see (1.8)). Note that the choice of $r=1 /|\log \varepsilon|^{\alpha}$ for the size of the balls $B_{i}$ is dictated by the fact that it is the largest radius for which we can prove that the expansion (1.8) is valid.

Of course, the structure of global minimizers for $h_{e x}>H_{c_{1}}$ is still open. We study them in [11], give an estimate on their energy, and 
we expect that the number of vortices is equivalent to $h_{e x}$ as soon as $h_{\text {ex }} \gg H_{c_{1}}$.

\section{PROOF OF THE THEOREM}

\subsection{Preliminary results and notations}

We consider $(u, A)$ such that

$$
J(u, A)=\inf \left\{J(v, B) \mid(v, B) \in H^{1}(\Omega, \mathbb{C}) \times H^{1}\left(\Omega, \mathbb{R}^{2}\right)\right\} .
$$

By a standard argument, this infimum is achieved and yields a solution of the Ginzburg-Landau equations. We recall that, as in [13], for a suitable choice of gauge, $\operatorname{div} A=0$, and there is a function $\xi \in H^{2}(\Omega, \mathbb{R})$ such that

$$
A=\nabla^{\perp} \xi=\left(-\xi_{x_{2}}, \xi_{x_{1}}\right), \quad \xi=0 \text { on } \partial \Omega,
$$

thus

$$
h=\Delta \xi,
$$

and

$$
\left|\nabla_{A} u\right|^{2}=|\nabla u|^{2}-2\left(i u, \xi_{x_{2}} u_{x_{1}}-\xi_{x_{1}} u_{x_{2}}\right)+|u|^{2}|A|^{2} .
$$

Our solution $(u, A)$ is easily seen to satisfy

$$
\left\{\begin{aligned}
\frac{\partial u}{\partial n} & =0 & & \text { on } \partial \Omega, \\
h & =h_{e x} & & \text { on } \partial \Omega .
\end{aligned}\right.
$$

In the sequel, $C$ denotes any positive constant independent from $\varepsilon$.

Since the value of $H_{c_{1}}$ computed in [13] is of the order of $|\log \varepsilon|$, we will assume from now on that

$$
h_{e x} \leqslant C|\log \varepsilon| .
$$

Considering the London equation (1.3), we are led as in [13] to introduce $\xi_{0}$, the solution of

$$
\left\{\begin{aligned}
-\Delta^{2} \xi_{0}+\Delta \xi_{0}=0 & \text { in } \Omega, \\
\Delta \xi_{0}=1 & \text { on } \partial \Omega, \\
\xi_{0}=0 & \text { on } \partial \Omega .
\end{aligned}\right.
$$


The approximate minimal vortex-less configuration is $\left(u_{0} \equiv 1, A_{0}=\right.$ $\left.h_{e x} \nabla^{\perp} \xi_{0}\right)$ and, as in [13], we let

$$
J_{0}=J\left(u_{0}, A_{0}\right)=-\frac{h_{e x}^{2}}{2} \int_{\Omega}\left|\nabla \xi_{0}\right|^{2}+\left|\Delta \xi_{0}\right|^{2} .
$$

Note that $\left(u_{0}, A_{0}\right)$ is only a solution to the second (G.L.) equation and not to the first one, therefore it is not the Meissner solution. However, it is proved in [13] that the infimum of the energy among vortex-less configurations in $D$ is $J_{0}+\mathrm{o}(1)$ as $\varepsilon \rightarrow 0$.

As in [13], we decompose $\xi$ as

$$
\xi=h_{e x} \xi_{0}+\zeta
$$

so that

$$
\zeta=0, \quad \Delta \zeta=0 \quad \text { on } \partial \Omega .
$$

We state some results borrowed from [13] that are going to be useful in the sequel.

LeMma 2.1. - Let $(u, A)$ be a solution of (G.L.). The following holds:

$$
\begin{gathered}
\|\nabla u\|_{L^{\infty}(\Omega)} \leqslant \frac{C}{\varepsilon}, \\
\|\nabla \xi\|_{L^{\infty}(\Omega)} \leqslant C h_{e x} .
\end{gathered}
$$

If $(u, A)$ is in addition a minimizer of the energy, then

$$
\begin{gathered}
J(u, A) \leqslant J_{0} \leqslant C h_{e x}^{2}, \\
\|\nabla u\|_{L^{2}(\Omega)} \leqslant C h_{e x} .
\end{gathered}
$$

Proof. - All the assertions have been proved in [13] except the last one. In view of (1.1) and (2.9),

$$
\int_{\Omega}\left|\nabla_{A} u\right|^{2} \leqslant C h_{e x}^{2}
$$

which is equivalent to

$$
\int_{\Omega}|\nabla u|^{2}-2\left(i u, \xi_{x_{2}} u_{x_{1}}-\xi_{x_{1}} u_{x-2}\right)+|u|^{2}|A|^{2} \leqslant C h_{e x}^{2} .
$$


Hence,

$$
\int_{\Omega}|\nabla u|^{2} \leqslant C h_{e x}^{2}+C\|\nabla \xi\|_{L^{\infty}(\Omega)}^{2}+C\|\nabla \xi\|_{L^{\infty}(\Omega)}\|\nabla u\|_{L^{2}(\Omega)} .
$$

Thus, in view of (2.8),

$$
\|\nabla u\|_{L^{2}(\Omega)} \leqslant C h_{e x} .
$$

We then need to define the vortices of $u$ with their degrees, by defining balls $\left(B_{i}\right)_{i \in I}$, such that $|u| \geqslant 3 / 4$ on $\Omega \backslash \bigcup_{i \in I} B_{i}$, and $d_{i}=\operatorname{deg}\left(u, \partial B_{i}\right)$. As already mentioned, we achieve this by adjusting a result of Jerrard [7], to obtain the following proposition, the proof of which is deffered to Section 3.

Proposition 2.1. - Let $u \in H^{1}(\Omega, \mathbb{C})$ such that $\|\nabla u\|_{L^{\infty}} \leqslant C / \varepsilon$, and $F(u) \leqslant C h_{e x}^{2}$. Then, for any $\alpha>0$, there is an $\varepsilon_{0}>0$ such that $\forall \varepsilon<$ $\varepsilon_{0}$ there exists a finite family of disjoint balls $\left(B_{i}\right)_{i \in I}=\left(B\left(a_{i}, r_{i}\right)\right)_{i \in I}$ such that

$$
\begin{gathered}
\left\{x /|u(x)|<\frac{3}{4}\right\} \subset \bigcup_{i \in I} B_{i}, \\
F_{\varepsilon}\left(u, B_{i}\right) \geqslant \pi\left|d_{i}\right|(|\log \varepsilon|-\mathrm{O}(|\log | \log \varepsilon||)),
\end{gathered}
$$

where $d_{i}=\operatorname{deg}\left(u, \partial B_{i}\right)$ if $\overline{B_{i}} \subset \Omega$, and $d_{i}=0$ otherwise,

$$
\begin{aligned}
& r_{i} \leqslant C|\log \varepsilon|^{-\alpha}, \\
& \text { Card } I \leqslant C h_{e x}^{2} .
\end{aligned}
$$

Proof. - See Proposition 3.2.

\subsection{Splitting of the energy}

Let

$$
\widetilde{\Omega}=\Omega \backslash \bigcup_{i \in I} B_{i},
$$

where $\left\{B_{i}\right\}_{i \in I}$ is the family of balls given by Proposition 2.1. Recall that they have radii $r_{i}$ less than $C|\log \varepsilon|^{-\alpha}$, where $\alpha$ is to be chosen below. 
LEMMA 2.2. - We have following identity:

$$
\begin{aligned}
\frac{1}{2} \int_{\Omega}\left|\nabla_{A} u\right|^{2}= & \int_{\Omega}\left(\frac{1}{2}\left|\nabla u-i \nabla^{\perp} \zeta u\right|^{2}+\frac{1}{2} h_{e x}^{2}\left|\nabla \xi_{0}\right|^{2}+h_{e x} \nabla \xi_{0} \cdot \nabla \zeta\right) \\
& +2 \pi h_{e x} \sum_{i \in I} d_{i} \xi_{0}\left(a_{i}\right)+\mathrm{o}(1) .
\end{aligned}
$$

Proof. - From (2.2) and (2.6),

$$
\begin{aligned}
|\nabla u-i A u|^{2}= & \left|\nabla u-i h_{e x} \nabla^{\perp} \xi_{0} u-i \nabla^{\perp} \zeta u\right|^{2} \\
= & \left|\nabla u-i \nabla^{\perp} \zeta u\right|^{2}+h_{e x}^{2}|u|^{2}\left|\nabla \xi_{0}\right|^{2} \\
& +2\left(\nabla u-i \nabla^{\perp} \zeta u,-i h_{e x} \nabla^{\perp} \xi_{0} u\right) .
\end{aligned}
$$

Moreover,

$$
\begin{aligned}
& \int_{\Omega}\left(\nabla u-i \nabla^{\perp} \zeta u,-i h_{e x} \nabla^{\perp} \xi_{0} u\right) \\
& \quad=\int_{\Omega}\left(\nabla u,-i h_{e x} \nabla^{\perp} \xi_{0} u\right)+h_{e x} \int_{\Omega}|u|^{2} \nabla \xi_{0} . \nabla \zeta .
\end{aligned}
$$

To finish the proof of Lemma 2.2 we need the

LEMMA 2.3. - If $\alpha>5$,

$$
\int_{\Omega}\left(\nabla u,-i h_{e x} \nabla^{\perp} \xi_{0} u\right)=2 \pi h_{e x} \sum_{i \in I} d_{i} \xi_{0}\left(a_{i}\right)+\mathrm{o}(1) .
$$

Proof. - We start with the same method as in [13]. First,

$$
\left|\int_{B_{i}}\left(\nabla u,-i h_{e x} \nabla^{\perp} \xi_{0} u\right)\right| \leqslant(\operatorname{Card} I) h_{e x}\|\nabla u\|_{L^{2}} \max _{i \in I} r_{i} \leqslant C|\log \varepsilon|^{4-\alpha},
$$

where we used (2.3), (2.13), (2.14), and (2.10). It is here that the size of the balls $B_{i}$ is important. Then, letting $\widetilde{\Omega}=\Omega \backslash \bigcup_{i} B_{i}$,

$$
\int_{\widetilde{\Omega}}\left(\nabla u,-i h_{e x} \nabla^{\perp} \xi_{0} u\right)=h_{e x} \int_{\widetilde{\Omega}}\left(i u,\left(\xi_{0}\right)_{x_{2}} u_{x_{1}}-\left(\xi_{0}\right)_{x_{1}} u_{x_{2}}\right) .
$$

Setting $v=u /|u|$ and integrating by parts, we find, exactly as in the proof of Lemma IV.3 of [13], that 


$$
\begin{aligned}
\int_{\widetilde{\Omega}} & \left(i u,\left(\xi_{0}\right)_{x_{2}} u_{x_{1}}-\left(\xi_{0}\right)_{x_{1}} u_{x_{2}}\right) \\
= & \int_{\widetilde{\Omega}}\left(i v, d v \wedge d \xi_{0}\right)+\mathrm{o}\left(|\log \varepsilon|^{-1}\right) \\
= & \sum_{i \in I_{\partial B_{i}}} \xi_{0}\left(i v, \frac{\partial v}{\partial \tau}\right)+\mathrm{o}\left(|\log \varepsilon|^{-1}\right) .
\end{aligned}
$$

We claim that, letting $\mathcal{J}=\left\{i \in I / \overline{B_{i}} \subset \Omega\right\}$,

$$
\forall i \in \mathcal{J}, \quad h_{e x} \int_{\partial B_{i}} \xi_{0}\left(i v, \frac{\partial v}{\partial \tau}\right)=2 \pi h_{e x} d_{i} \xi_{0}\left(a_{i}\right)+\mathrm{o}\left(|\log \varepsilon|^{-2}\right) .
$$

To prove this claim, we use the same proof as in [13] (Lemma IV.3), different from that of [5] which does not adjust to a possible divergent number of balls. Let

$$
U_{i}=\left\{x \in B_{i} /|u| \leqslant \frac{1}{2}\right\} .
$$

$U_{i}$ does not intersect $\partial B_{i}$ and by Stokes' theorem

$$
\begin{aligned}
& \left|\int_{\partial B_{i}}\left(\xi_{0}-\xi_{0}\left(a_{i}\right)\right)\left(i v, \frac{\partial v}{\partial \tau}\right)-\int_{\partial U_{i}}\left(\xi_{0}-\xi_{0}\left(a_{i}\right)\right)\left(i v, \frac{\partial v}{\partial \tau}\right)\right| \\
& \quad=\left|\int_{B_{i} \backslash U_{i}} d \xi_{0} \wedge(i v, d v)\right| \leqslant C\left\|\nabla \xi_{0}\right\|_{L^{\infty}} r_{i}\left(\int_{B_{i} \backslash U_{i}}|\nabla v|^{2}\right)^{1 / 2} \\
& \quad \leqslant C|\log \varepsilon|^{1-\alpha} .
\end{aligned}
$$

Hence, as $\alpha>5$,

$$
\begin{aligned}
h_{e x} & \int_{\partial B_{i}}\left(\xi_{0}-\xi_{0}\left(a_{i}\right)\right)(i v, d v) \\
& =h_{e x} \int_{\partial U_{i}}\left(\xi_{0}-\xi_{0}\left(a_{i}\right)\right)(i v, d v)+\mathrm{o}\left(|\log \varepsilon|^{-2}\right) .
\end{aligned}
$$

On the other hand,

$$
h_{e x} \int_{\partial U_{i}}\left(\xi_{0}-\xi_{0}\left(a_{i}\right)\right)(i v, d v)=h_{e x} \int_{\partial U_{i}}\left(\xi_{0}-\xi_{0}\left(a_{i}\right)\right) \frac{(i u, d u)}{|u|^{2}} .
$$


But, by definition of $U_{i},|u|=1 / 2$ on $\partial U_{i}$, hence

$$
\begin{aligned}
& h_{e x}\left|\int_{\partial U_{i}}\left(\xi_{0}-\xi_{0}\left(a_{i}\right)\right)(i v, d v)\right| \\
& \quad=4 h_{e x}\left|\int_{\partial U_{i}}\left(\xi_{0}-\xi_{0}\left(a_{i}\right)\right)(i u, d u)\right| \\
& \quad=4 h_{e x}\left|\int_{U_{i}} d \xi_{0} \wedge(i u, d u)+\left(\xi_{0}-\xi_{0}\left(a_{i}\right)\right)(i d u, d u)\right| \\
& \quad \leqslant C h_{e x} r_{i}\|\nabla u\|_{L^{2}}+C h_{e x} r_{i}\left\|\nabla \xi_{0}\right\|_{L^{\infty}} \int_{\Omega}|\nabla u|^{2} \\
& \quad \leqslant C|\log \varepsilon|^{3-\alpha} \leqslant \mathrm{o}\left(|\log \varepsilon|^{-2}\right) .
\end{aligned}
$$

Combining (2.20) and (2.21), we conclude that

$$
\begin{aligned}
h_{e x} \int_{\partial B_{i}} \xi_{0}(i v, d v) & =h_{e x} \int_{\partial B_{i}} \xi_{0}\left(a_{i}\right)(i v, d v)+\mathrm{o}\left(|\log \varepsilon|^{-2}\right) \\
& =2 \pi h_{e x} d_{i} \xi_{0}\left(a_{i}\right)+\mathrm{o}\left(|\log \varepsilon|^{-2}\right),
\end{aligned}
$$

and (2.19) is proved.

We now deal with the balls that intersect $\partial \Omega$. We claim that

$$
\forall i \in I \backslash \mathcal{J}, \quad h_{e x} \int_{\partial B_{i} \cap \Omega} \xi_{0}(i v, d v)=\mathrm{o}\left(|\log \varepsilon|^{-2}\right) .
$$

The proof of this claim is almost the same as that of (2.19). Indeed, since $\xi_{0}=0$ on $\partial \Omega$, letting $U_{i}=B_{i} \cap\{|u| \leqslant 1 / 2\}$,

$$
\begin{aligned}
h_{e x} & \int_{\partial B_{i} \cap \Omega} \xi_{0}(i v, d v) \\
= & h_{e x} \int_{\partial U_{i} \cap \Omega} \xi_{0}(i v, d v)+\mathrm{o}\left(|\log \varepsilon|^{-2}\right) \\
= & 4 h_{e x}\left|\int_{U_{i} \cap \Omega} d \xi_{0} \wedge(i u, d u)+\xi_{0}(i d u, d u)\right|=\mathrm{o}\left(|\log \varepsilon|^{-2}\right) .
\end{aligned}
$$

Using (2.18) and (2.19), the above claim and the fact that Card $I \leqslant C h_{e x}^{2}$ prove the lemma. 
Proof of Lemma 2.2 completed. - Combining (2.15), (2.16) and (2.17), we are led to

$$
\begin{aligned}
\frac{1}{2} \int_{\Omega}\left|\nabla_{A} u\right|^{2}= & \frac{1}{2} \int_{\Omega}\left|\nabla u-i \nabla^{\perp} \zeta u\right|^{2}+\frac{1}{2} h_{e x}^{2} \int_{\Omega}|u|^{2}\left|\nabla \xi_{0}\right|^{2} \\
& +h_{e x} \int_{\Omega}|u|^{2} \nabla \xi_{0} . \nabla \zeta+2 \pi h_{e x} \sum_{i \in I} d_{i} \xi_{0}\left(a_{i}\right)+\mathrm{o}(1) .
\end{aligned}
$$

From the upper bound (2.9), we know that

$$
\frac{1}{\varepsilon^{2}} \int_{\Omega}\left(1-|u|^{2}\right)^{2} \leqslant C h_{e x}^{2},
$$

hence

$$
\begin{aligned}
h_{e x}^{2} \int_{\Omega}\left(1-|u|^{2}\right)\left|\nabla \xi_{0}\right|^{2} & \leqslant C h_{e x}^{2}\left(\int_{\Omega}\left(1-|u|^{2}\right)^{2}\right)^{1 / 2} \\
& \leqslant C \varepsilon h_{e x}^{3} \leqslant \mathrm{o}(1),
\end{aligned}
$$

and similarly

$$
h_{e x}^{2}\left|\int_{\Omega}\left(1-|u|^{2}\right) \nabla \xi_{0} . \nabla \zeta\right| \leqslant C \varepsilon h_{e x}^{3} \leqslant \mathrm{o}(1) .
$$

With (2.22), the lemma is proved.

LEMMA 2.4. - We have the following identity:

$$
\begin{aligned}
& \frac{1}{2} \int_{\Omega}\left(\left|h-h_{e x}\right|^{2}-h_{e x}^{2}\right)+\frac{1}{2} h_{e x}^{2} \int_{\Omega}\left|\nabla \xi_{0}\right|^{2}+h_{e x} \int_{\Omega} \nabla \xi_{0} . \nabla \zeta \\
& \quad=J_{0}+\int_{\Omega}|\Delta \zeta|^{2} .
\end{aligned}
$$

Proof. - Using the decomposition (2.6),

$$
\begin{aligned}
\int_{\Omega} & \left(\left|h-h_{e x}\right|^{2}-h_{e x}^{2}\right) \\
= & \int_{\Omega}\left|h_{e x} \Delta \xi_{0}+\Delta \zeta\right|^{2}-2 h_{e x}\left(h_{e x} \Delta \xi_{0}+\Delta \zeta\right)
\end{aligned}
$$




$$
\begin{aligned}
= & h_{e x}^{2} \int_{\Omega}\left|\Delta \xi_{0}\right|^{2}+\int_{\Omega}|\Delta \zeta|^{2}-2 h_{e x}^{2} \int_{\Omega} \Delta \xi_{0}+2 h_{e x} \int_{\Omega} \Delta \xi_{0} \Delta \zeta \\
& -2 h_{e x} \int_{\Omega} \Delta \zeta .
\end{aligned}
$$

But, from [13], Section 4 (using (2.4)),

$$
\int_{\Omega}\left|\nabla \xi_{0}\right|^{2}+\left|\Delta \xi_{0}\right|^{2}=\int_{\Omega} \Delta \xi_{0},
$$

and

$$
\int_{\Omega} \nabla \zeta . \nabla \xi_{0}+\Delta \zeta \Delta \xi_{0}=\int_{\Omega} \Delta \zeta
$$

Therefore,

$$
\begin{aligned}
& \frac{1}{2} \int_{\Omega}\left(\left|h-h_{e x}\right|^{2}-h_{e x}^{2}\right)+\frac{1}{2} h_{e x}^{2} \int_{\Omega}\left|\nabla \xi_{0}\right|^{2}+h_{e x} \int_{\Omega} \nabla \xi_{0} . \nabla \zeta \\
& \quad=-\frac{h_{e x}^{2}}{2} \int_{\Omega}\left|\nabla \xi_{0}\right|^{2}+\left|\Delta \xi_{0}\right|^{2}+\int_{\Omega}|\Delta \zeta|^{2} \\
& \quad=J_{0}+\int_{\Omega}|\Delta \zeta|^{2} .
\end{aligned}
$$

This completes the proof.

Combining Lemmas 2.2 and 2.4, we obtain the following expansion of the energy:

$$
\begin{aligned}
J(u, A)= & \frac{1}{2} \int_{\Omega}\left|\nabla u-i u \nabla^{\perp} \zeta\right|^{2}+\frac{1}{2 \varepsilon^{2}}\left(1-|u|^{2}\right)^{2}+\int_{\Omega}|\Delta \zeta|^{2} \\
& +J_{0}+2 \pi h_{e x} \sum_{i \in I} d_{i} \xi_{0}\left(a_{i}\right)+o(1)
\end{aligned}
$$

Notice that this expansion is quite similar to that of [13], but the terms in $\zeta$ are treated differently and gathered in positive expressions.

We need a last lower bound:

LEMMA 2.5. -

$$
\int_{\Omega}\left|\nabla u-i u \nabla^{\perp} \zeta\right|^{2} \geqslant \sum_{i \in I} \int_{B_{i}}|\nabla u|^{2}+\mathrm{o}(1) .
$$


Proof. - Indeed,

$$
\begin{aligned}
\int_{\Omega}\left|\nabla u-i u \nabla^{\perp} \zeta\right|^{2} & \geqslant \int_{\bigcup_{i \in I} B_{i}}\left|\nabla u-i u \nabla^{\perp} \zeta\right|^{2} \\
& \geqslant \int_{\bigcup_{i \in I} B_{i}}|\nabla u|^{2}+\left|u \nabla^{\perp} \zeta\right|^{2}-2\left(\nabla u, i u \nabla^{\perp} \zeta\right) .
\end{aligned}
$$

But,

$$
\begin{aligned}
\left|\int_{i \in I}\left(\nabla u, i u \nabla^{\perp} \zeta\right)\right| & \leqslant(\operatorname{Card} I)\|\nabla \xi\|_{L^{\infty}}\|\nabla u\|_{L^{2}} \max _{i} r_{i} \\
& \leqslant C|\log \varepsilon|^{4-\alpha}=\mathrm{o}(1)
\end{aligned}
$$

similarly to the beginning of the proof of Lemma 2.3. Hence,

$$
\int_{\Omega}\left|\nabla u-i u \nabla^{\perp} \zeta\right|^{2} \geqslant \int_{\bigcup_{i \in I} B_{i}}|\nabla u|^{2}+\mathrm{o}(1) .
$$

From this lemma, we deduce that

$$
\begin{aligned}
& \frac{1}{2} \int_{\Omega}\left|\nabla u-i u \nabla^{\perp} \zeta\right|^{2}+\frac{1}{2 \varepsilon^{2}}\left(1-|u|^{2}\right)^{2} \\
& \geqslant \sum_{i \in I} \int_{B_{i}} \frac{1}{2}|\nabla u|^{2}+\frac{1}{4 \varepsilon^{2}}\left(1-|u|^{2}\right)^{2} \\
& \geqslant \sum_{i \in I} F\left(u, B_{i}\right),
\end{aligned}
$$

and this last expression can be bounded from below by (2.12).

\subsection{End of the proof of the theorem}

Considering our minimizing solution, we deduce from (2.24), Lemma 2.5 , and (2.12), that

$$
\begin{aligned}
J(u, A) \geqslant & J_{0}+2 \pi h_{e x} \sum_{i \in I} d_{i} \xi_{0}\left(a_{i}\right) \\
& +\pi \sum_{i \in I}\left|d_{i}\right|(|\log \varepsilon|+\mathrm{O}(|\log | \log \varepsilon||)) .
\end{aligned}
$$


On the other hand, by minimality,

$$
J(u, A) \leqslant J_{0},
$$

thus, as $\xi_{0}$ is negative,

$$
\begin{aligned}
\pi \sum_{i \in I}\left|d_{i}\right|(|\log \varepsilon|+\mathrm{O}(|\log | \log \varepsilon||)) & \leqslant 2 \pi h_{e x} \sum_{i \in I} d_{i}\left|\xi_{0}\left(a_{i}\right)\right| \\
& \leqslant 2 \pi h_{e x}\left(\sum_{i \in I}\left|d_{i}\right|\right) \max \left|\xi_{0}\right| .
\end{aligned}
$$

If $\sum_{i \in I}\left|d_{i}\right| \neq 0$, we deduce that

$$
h_{e x} \geqslant \frac{1}{2 \max \left|\xi_{0}\right|}(|\log \varepsilon|+\mathrm{O}(|\log | \log \varepsilon||)):=H_{c_{1}}^{\prime} .
$$

But, in [13], $H_{c_{1}}=k_{1}|\log \varepsilon|+\mathrm{O}(1)$ with

$$
k_{1}=\frac{1}{2 \max \left|\xi_{0}\right|}
$$

thus

$$
H_{c_{1}}^{\prime}=H_{c_{1}}+\mathrm{O}(|\log | \log \varepsilon||) .
$$

Consequently, if $h_{e x}<H_{c_{1}}^{\prime}$, we must have $d_{i}=0, \forall i \in I$. Then, with (2.24) and Lemma 2.5,

$$
J_{0} \geqslant J(u, A) \geqslant \sum_{i \in I} F_{\varepsilon}\left(u, B_{i}\right)+J_{0}+\mathrm{o}(1),
$$

implying

$$
\sum_{i \in I} F_{\varepsilon}\left(u, B_{i}\right) \leqslant \mathrm{o}(1) .
$$

We conclude that $|u| \geqslant 3 / 4$ in $\Omega$. Indeed, it is well known from [3] that if $\left|u\left(x_{0}\right)\right|<3 / 4$, there exist constants $\lambda, \mu>0$ such that

$$
\forall \varepsilon>0, \quad \frac{1}{\varepsilon^{2}} \int_{B\left(x_{0}, \lambda \varepsilon\right)}\left(1-|u|^{2}\right)^{2}>\mu>0,
$$

contradicting (2.28). 
Knowing that $u$ is vortex-less, one may re-use the computations of [13] to find that

$$
J(u, A)=J_{0}+F(u)+\widetilde{V}(\zeta)+\mathrm{o}(1),
$$

where $\widetilde{V}(\zeta) \geqslant 0$. Hence $F(u) \leqslant \mathrm{o}(1)$, and by definition $(u, A) \in D$. This proves that for $h_{e x}<H_{c_{1}}^{\prime},(u, A)$ coincides with the unique Meissner solution found in [13] and [15].

The theorem is proved.

\section{CONSTRUCTION OF THE BALLS}

In this section, we use the method of R. Jerrard introduced in [7], in order to construct balls containing all the zeros of $u$, on which we have a suitable lower bound on $F_{\varepsilon}$ of the order $\pi|d||\log \varepsilon|$. The size of the balls has to be large enough so that most of the energy $F_{\varepsilon}$ is concentrated in these balls, but it has to be smaller than $|\log \varepsilon|^{-5}$ as we saw in Section 2 . We follow almost readily the proofs of [7].

\subsection{Main steps}

First, we include the set $\{x /|u(x)|<3 / 4\}$ in well-chosen disjoint "small" balls $B_{i}$ of radii $r_{i}>\varepsilon$ such that

$$
F_{\varepsilon}\left(u, B_{i}\right) \geqslant \frac{C_{1} r_{i}}{\varepsilon},
$$

where $C_{1}$ is a constant. This is possible according to the following lemma, adjusted from [7]:

Lemma 3.1. - Let $u: \Omega \rightarrow \mathbb{C}$ be such that $|\nabla u|<C / \varepsilon$. Then there exist disjoint balls $B_{1}, \ldots, B_{k}$ of radii $r_{i}$ such that

(1) $\forall 1 \leqslant i \leqslant k, r_{i}>\varepsilon$,

(2) $\{|u|<3 / 4\} \subset \bigcup_{i} B_{i}$ and $\forall 1 \leqslant i \leqslant k, B_{i} \cap\{|u|<3 / 4\} \neq \emptyset$,

(3) $\forall 1 \leqslant i \leqslant k$,

$$
F_{\varepsilon}\left(u, B_{i} \cap \Omega\right) \geqslant \frac{C_{1} r_{i}}{\varepsilon} .
$$

Then the proof involves dilating the balls $B_{i}$ into balls $B_{i}^{\prime}$. A lower bound for $F_{\varepsilon}\left(u, B_{i}^{\prime}\right)$ is obtained by combining the lower bound for $F_{\varepsilon}\left(u, B_{i}\right)$ and a lower bound of the energy on the annulus $B_{i}^{\prime} \backslash \overline{B_{i}}$ : 
LEMMA 3.2. $-\forall r>s>\varepsilon$, if $B_{r}$ and $B_{s}$ are two concentric balls of respective radii $r$ and $s$, and if $u: B_{r} \backslash \overline{B_{s}} \rightarrow \mathbb{C}$ is such that $|u|>3 / 4, d=$ $\operatorname{deg}\left(u, \partial B_{r}\right)$, then

$$
F_{\varepsilon}\left(u, B_{r} \backslash \overline{B_{s}}\right) \geqslant|d|\left(\Lambda_{\varepsilon}\left(\frac{r}{|d|}\right)-\Lambda_{\varepsilon}\left(\frac{s}{|d|}\right)\right) \text {, }
$$

where $\Lambda_{\varepsilon}$ is a function that satisfies the following properties:

(1) $\Lambda_{\varepsilon}(s) / s$ is decreasing on $\mathbb{R}_{+}$,

(2) $\sup _{s \in \mathbb{R}_{+}} \Lambda_{\varepsilon}(s) / s \leqslant C_{1} / \varepsilon$,

(3) there exist $\varepsilon_{0}, t_{0}>0$ such that if $\varepsilon<\varepsilon_{0}$ and $\varepsilon<t<t_{0}$ then

$$
\left|\Lambda_{\varepsilon}(t)-\pi \log \frac{t}{\varepsilon}\right| \leqslant C .
$$

Thanks to this lemma, if

$$
F_{\varepsilon}(u, B) \geqslant \pi|d| \log \frac{r}{\varepsilon}
$$

where $d=\operatorname{deg}(u, \partial B)$, and $r$ is the radius of $B$, if $B^{\prime}$ is the dilated ball, and if $|u| \geqslant 3 / 4$ on $B_{i}^{\prime} \backslash \overline{B_{i}}$, then an estimate of the type (3.2) is still true on $B^{\prime}$.

Thus we start with the balls $B_{i}$ given by Lemma 3.1, then make them grow progressively. Say the growth rate is governed by a parameter $s$, we thus construct a family $\mathcal{B}(s)$ of disjoint balls. To keep this family of balls disjoint, when some of them intersect, we merge them into a larger ball of radius equal to the sum of the radii of the merged balls. If the growthrates of the balls have been properly synchronized, then the lower bounds for the energy on each of the merged balls add up nicely so that a lower bound of the type (3.2) is still true for the larger ball. We then resume the dilation, etc., until we reach the size of balls that we wish.

The following proposition sums up the whole growth process:

Proposition 3.1. - Let $u: \Omega \rightarrow \mathbb{C}$ be such that $|\nabla u| \leqslant C / \varepsilon$; and $\left\{B_{i}\right\}_{i}$ be a family of balls of radii $r_{i}$ satisfying the results of Lemma 3.1. Let

$$
d_{i}= \begin{cases}\operatorname{deg}\left(u, \partial B_{i}\right) & \text { if } \overline{B_{i}} \subset \Omega \\ 0 & \text { otherwise }\end{cases}
$$


Let also

$$
s_{0}=\min _{\left\{i \mid d_{i} \neq 0\right\}} \frac{r_{i}}{\left|d_{i}\right|} .
$$

Then, for every $s \geqslant s_{0}$, there exists a family $\mathcal{B}(s)$ of disjoint balls $B_{1}(s), \ldots, B_{k(s)}(s)$ of radii $r_{i}(s)$ such that

(1) the family of balls is monotone, i.e., if $s<t$ then

$$
\bigcup_{i} B_{i}(s) \subset \bigcup_{i} B_{i}(t)
$$

(2) for every $i, F_{\varepsilon}\left(u, B_{i}(s)\right) \geqslant r_{i}(s) \Lambda_{\varepsilon}(s) / s$, where $\Lambda_{\varepsilon}$ is defined in Lemma 3.2,

(3) if $\overline{B_{i}(s)} \subset \Omega$ and $d_{i}(s)=\operatorname{deg}\left(u, \partial B_{i}(s)\right)$, then $r_{i}(s) \geqslant s\left|d_{i}(s)\right|$.

We then get as a consequence the following proposition, that was stated as Proposition 2.1 in Section 2, and which yields the final balls that we needed:

Proposition 3.2. - Let $u: \Omega \rightarrow \mathbb{C}$ be such that $|\nabla u| \leqslant C / \varepsilon$ and that $F_{\varepsilon}(u) \leqslant C|\log \varepsilon|^{2}$. Then, for any $\alpha>0$ there exist disjoint balls $\left(B_{i}\right)_{i \in I}$ of radii $r_{i}$ such that, for sufficiently small $\varepsilon$,

(1) $\{x /|u(x)|<3 / 4\} \subset \bigcup_{i \in I} B_{i}$,

(2) $\operatorname{Card} I \leqslant C|\log \varepsilon|^{2}$,

(3) $r_{i} \leqslant C /|\log \varepsilon|^{\alpha}$,

(4) if $\overline{B_{i}} \subset \Omega$ and $d_{i}=\operatorname{deg}\left(u, \partial B_{i}\right)$, then

$$
F_{\varepsilon}\left(u, B_{i}\right) \geqslant \pi\left|d_{i}\right|(|\log \varepsilon|-\mathrm{O}(|\log | \log \varepsilon||)) .
$$

Proof. - We first consider the balls given by Lemma 3.1, and then apply Proposition 3.1 to get bigger balls. We need to check that $s_{0}$ is small enough to be able to apply Proposition 3.1 for $s$ large enough. Indeed, $s_{0}=\min _{\left\{i \mid d_{i} \neq 0\right\}}\left(r_{i} / d_{i}\right)$, but from assertion (3) of Lemma 3.1,

$$
C_{1} r_{i}<\varepsilon F_{\varepsilon}\left(u, B_{i} \cap \Omega\right) \leqslant C \varepsilon|\log \varepsilon|^{2},
$$

so that $s_{0} \leqslant C \varepsilon|\log \varepsilon|^{2}$. We can thus apply Proposition 3.1 for all $s \geqslant$ $C \varepsilon|\log \varepsilon|^{2}$. We choose in particular

$$
s_{1}=\frac{1}{|\log \varepsilon|^{\alpha+1}} .
$$


Proposition 3.1 yields final balls $\mathcal{B}\left(s_{1}\right)$ such that

$$
\forall i, \text { if } \overline{B_{i}\left(s_{1}\right)} \subset \Omega, \quad F_{\varepsilon}\left(u, B_{i}\left(s_{1}\right)\right) \geqslant \frac{\Lambda_{\varepsilon}\left(s_{1}\right)}{s_{1}} r_{i}\left(s_{1}\right),
$$

with

$$
r_{i}\left(s_{1}\right) \geqslant s_{1}\left|d_{i}\left(s_{1}\right)\right|
$$

Therefore,

$$
F_{\varepsilon}\left(u, B_{i}\left(s_{1}\right)\right) \geqslant \Lambda_{\varepsilon}\left(s_{1}\right)\left|d_{i}\left(s_{1}\right)\right|,
$$

and from Lemma 3.2 (assertion (3)),

$$
\begin{aligned}
F_{\varepsilon}\left(u, B_{i}\left(s_{1}\right)\right) & \geqslant\left|d_{i}\left(s_{1}\right)\right|\left(\pi \log \frac{s_{1}}{\varepsilon}-C\right) \\
& \geqslant \pi\left|d_{i}\left(s_{1}\right)\right|(|\log \varepsilon|-\mathrm{O}(|\log | \log \varepsilon||)) .
\end{aligned}
$$

We thus have the lower bound (4) on $F_{\varepsilon}$.

We then show that (3) is true. We know that $F_{\varepsilon}(u) \leqslant C h_{e x}^{2} \leqslant C|\log \varepsilon|^{2}$. Combining this with (3.5), we get

$$
r_{i}\left(s_{1}\right) \frac{\Lambda_{\varepsilon}\left(s_{1}\right)}{s_{1}} \leqslant C|\log \varepsilon|^{2} .
$$

But, as $\Lambda_{\varepsilon}(s) \simeq \pi \log (s / \varepsilon)$, in view of (3.4),

$$
\Lambda_{\varepsilon}\left(s_{1}\right) \geqslant \pi|\log \varepsilon|-\mathrm{O}(|\log | \log \varepsilon||) .
$$

Hence, if $\varepsilon$ is sufficiently small,

$$
r_{i}\left(s_{1}\right) \leqslant C \frac{s_{1}}{\Lambda_{\varepsilon}\left(s_{1}\right)}|\log \varepsilon|^{2} \leqslant \frac{C}{|\log \varepsilon|^{\alpha+1}} \frac{|\log \varepsilon|^{2}}{|\log \varepsilon|} \leqslant \frac{C}{|\log \varepsilon|^{\alpha}},
$$

which is the desired estimate.

There only remains to show that (2) is true. This is easy since in Lemma 3.1, each ball satisfies $F_{\varepsilon}\left(u, B_{i} \cap \Omega\right) \geqslant C_{1} r_{i} / \varepsilon$, with $r_{i}>\varepsilon$, hence carries an energy that is bounded from below by a constant independent from $\varepsilon$. As $F_{\varepsilon} \leqslant C|\log \varepsilon|^{2}$, we see that the number of these balls has to be bounded by $C|\log \varepsilon|^{2}$. Then, the procedure of Proposition 3.1 does not increase the number of balls, so that property (2) is true. 


\subsection{Proof of Lemma 3.1}

We use the notation $S(x, r)$ for the circle in $\mathbb{R}^{2}$ of center $x$ and radius $r$. We begin with the following lemma, taken from [7]:

Lemma 3.3. - Let $u: S_{t} \rightarrow \mathbb{C}$, where $S_{t}$ is a circle of radius $t$ in $\mathbb{R}^{2}$ such that $t>\varepsilon$. Let $m=\min _{S_{t}}|u|$ and, if $m \neq 0, d=\operatorname{deg}\left(u, S_{t}\right)$. If $m=0$, let $d=0$. Then, assuming $0 \leqslant m \leqslant 1$,

$$
\int_{S_{t}} \frac{1}{2}|\nabla u|^{2}+\frac{1}{4 \varepsilon^{2}}\left(1-|u|^{2}\right)^{2} \geqslant m^{2} \frac{\pi d^{2}}{t}+\frac{(1-m)^{C_{2}}}{C_{2} \varepsilon},
$$

where $C_{2}$ is an absolute constant.

Proof. - See [7], Theorem 2.1.

We also have the following variant of Lemma 3.3 (Lemma 2.4 in [7]):

LEMMA 3.4. - If $u: \Omega \rightarrow \mathbb{C}$, there exists $\rho(\Omega), C(\Omega)>0$ such that $\forall x \in \Omega, \forall \varepsilon<r<\rho$, letting $m=\min _{S_{r} \cap \Omega}|u|$,

$$
F_{\varepsilon}\left(u, S_{r}\right) \geqslant \frac{(1-m)^{C}}{C \varepsilon} .
$$

Proof. - See [7].

We then divide the proof of Lemma 3.1 in five steps.

Step 1. We wish to include $\{|u|<3 / 4\}$ in balls. Let $S_{1}, \ldots, S_{k}$ be the connected components of $\{|u|<4 / 5\}$ that intersect $\{|u|<3 / 4\}$, and $x_{1} \in S_{1}, \ldots, x_{k} \in S_{k}$ be such that $\left|u\left(x_{i}\right)\right|<3 / 4$. Also, for every $i$ let

$$
r_{i}=\sup \left\{r>0 \mid S\left(x_{i}, r\right) \cap\{|u|<4 / 5\} \neq \emptyset\right\} .
$$

We claim that

$$
F_{\varepsilon}\left(u, B\left(x_{i}, r_{i}\right) \cap \Omega\right) \geqslant \frac{C_{1} r_{i}}{\varepsilon} .
$$

Indeed from the hypothesis $|\nabla u|<C / \varepsilon$, we get as in (2.29) that $F_{\varepsilon}\left(u, B\left(x_{i}, r_{i}\right) \cap \Omega\right) \geqslant C$. Therefore if $r_{i} \leqslant 2 \varepsilon$, the claim is true.

If $r_{i}>2 \varepsilon$ then, from Lemma 3.4, as $\forall t \in\left[\varepsilon, r_{i}\right], \min _{S\left(x_{i}, t\right)}|u|<4 / 5$ by definition of $r_{i}$,

$$
\forall t \in\left[\varepsilon, r_{i}\right], \quad F_{\varepsilon}\left(u, S\left(x_{i}, t\right) \cap \Omega\right) \geqslant \frac{C}{\varepsilon} .
$$


We deduce that

$$
\begin{aligned}
F_{\varepsilon}\left(u, \Omega \cap B\left(x_{i}, r_{i}\right)\right) & \geqslant \int_{\varepsilon}^{r_{i}} F_{\varepsilon}\left(u, \Omega \cap S\left(x_{i}, t\right)\right) d t \\
& \geqslant \frac{C\left(r_{i}-\varepsilon\right)}{\varepsilon} \geqslant \frac{C r_{i}}{\varepsilon},
\end{aligned}
$$

proving the claim in this case also.

Step 2. For simplicity, we write $B_{i}$ for $B\left(x_{i}, r_{i}\right)$. We claim that $\forall i \neq j$, either $B_{j} \subset B_{i}$ (in this case, we drop $B_{j}$ ) or $x_{j} \notin B_{i}$.

Indeed, assume that $x_{j} \in B_{i}$. By definition of the balls $B_{i}, \partial B_{i} \cap$ $\bigcup_{\ell} S_{\ell}=\emptyset$, thus

$$
S_{j}=\left(S_{j} \cap \complement \overline{B_{i}}\right) \cup\left(S_{j} \cap B_{i}\right) .
$$

Since $x_{j} \in B_{i}, S_{j} \cap B_{i} \neq \emptyset$, using the connectedness of $S_{j}, S_{j} \subset B_{i}$, and we can $\operatorname{drop} B_{j}$.

The claim is proved.

Step 3. Dropping the unnecessary balls, we reduce to balls $B_{i}$ such that

$$
\bigcup_{i} S_{i} \subset \bigcup_{k} B_{k} \quad \text { and } \quad \forall i \neq j, x_{j} \notin B_{i}
$$

It follows from the Besicovitch covering lemma (see for instance [17], p. 44), any $x \in \bigcup B_{i}$ belongs to at most $N$ of the balls, where $N$ is an absolute constant.

Step 4. Naming $\mathcal{C}_{i}$ the connected components of $\bigcup_{i} B_{i}$, this implies that

$$
\begin{aligned}
\forall i \quad F_{\varepsilon}\left(u, \mathcal{C}_{i}\right) & \geqslant \frac{1}{N} \sum_{j / B_{j} \subset \mathcal{C}_{i}} F_{\varepsilon}\left(u, B_{j}\right) \geqslant C \sum_{j / B_{j} \subset \mathcal{C}_{i}} \frac{r_{j}}{\varepsilon} \\
& \geqslant C \frac{\operatorname{diam} \mathcal{C}_{i}}{\varepsilon},
\end{aligned}
$$

where we have used (3.9).

Step 5. Each $\mathcal{C}_{i}$ can be included in a ball $B_{i}^{\prime}$ of radius

$$
r_{i}^{\prime} \leqslant \operatorname{diam} \mathcal{C}_{i}
$$

These balls $B_{i}^{\prime}$ can be included in bigger balls $B_{i}^{\prime \prime}$ the following way: if $B_{i}^{\prime}$ and $B_{j}^{\prime}$ intersect, we merge them into a ball of radius no higher than $r_{i}^{\prime}+r_{j}^{\prime}$, etc., until all intersecting balls are merged. Hence, we are left 
with a family $B_{i}^{\prime \prime}$ of disjoint balls of radii $r_{i}^{\prime \prime}$. This family satisfies

$$
r_{i}^{\prime \prime} \leqslant \sum_{j / B_{j}^{\prime} \subset B_{i}^{\prime \prime}} r_{j}^{\prime}
$$

As the $\mathcal{C}_{i}$ are disjoint, we have

$$
F_{\varepsilon}\left(u, B_{i}^{\prime \prime}\right) \geqslant \sum_{j / \mathcal{C}_{j} \subset B_{i}^{\prime \prime}} F_{\varepsilon}\left(u, \mathcal{C}_{j}\right) \geqslant \sum_{j / \mathcal{C}_{j} \subset B_{i}^{\prime \prime}} C \frac{\operatorname{diam} \mathcal{C}_{j}}{\varepsilon},
$$

with (3.10). Using (3.11) and (3.12), we are led to

$$
F_{\varepsilon}\left(u, B_{i}^{\prime \prime}\right) \geqslant \sum_{j / \mathcal{C}_{j} \subset B_{i}^{\prime \prime}} C \frac{r_{j}^{\prime}}{\varepsilon} \geqslant C \frac{r_{i}^{\prime \prime}}{\varepsilon} .
$$

The family $\left(B_{i}^{\prime \prime}\right)$ satisfies the desired conditions, hence the proof is complete.

\subsection{Proof of Lemma 3.2}

Under the assumptions of Lemma 3.3,

$$
F_{\varepsilon}\left(u, S_{t}\right) \geqslant f_{\varepsilon}\left(\frac{t}{|d|}\right),
$$

where

$$
f_{\varepsilon}(s)=\frac{a b}{\left(a^{q}+b^{q}\right)^{1 / q}}, \quad a(s)=\pi / s, b(s)=\frac{C_{2}}{\varepsilon},
$$

and $0 \leqslant q \leqslant 1$ is a constant.

Indeed, using Lemma 3.3, we can bound the right-hand side of (3.7) from below by

$$
\left(m^{C^{\prime}}\right) \frac{\pi|d|}{t}+\frac{C_{2}(1-m)^{C^{\prime}}}{\varepsilon},
$$

where $C^{\prime}=\max \left(2, C_{2}\right) \geqslant 2$. This uses the fact that $0<m<1$. Then we minimize with respect to $m \in[0,1]$. This yields (3.9) with $q=$ $1 /\left(C^{\prime}-1\right)$.

We then define

$$
\lambda_{\varepsilon}(s)=\min \left(f_{\varepsilon}(s), \frac{C_{1}}{\varepsilon}\right)
$$


where $C_{1}$ is defined in Lemma 3.1 Also, we let

$$
\Lambda_{\varepsilon}(t)=\int_{0}^{t} \lambda_{\varepsilon}(s) d s
$$

We prove the properties on $\Lambda_{\varepsilon}$. (1) is true because $\Lambda_{\varepsilon}$ is the primitive of $\lambda_{\varepsilon}$ which is easily seen to be decreasing. From this, we deduce that

$$
\sup _{s \in \mathbb{R}_{+}} \frac{\Lambda_{\varepsilon}(s)}{s}=\lambda_{\varepsilon}(0) \leqslant \frac{C_{1}}{\varepsilon}
$$

so that (2) is true. There remains to prove assertion (3). Recall that

$$
\Lambda_{\varepsilon}(s)=\int_{0}^{s} \frac{a}{\left((a / b)^{q}+1\right)^{1 / q}} d t, \quad \text { where } a=\frac{\pi}{t}, b=\frac{C_{2}}{\varepsilon} .
$$

Denoting by $C, C^{\prime}$ generic positive constants, if $C$ is large enough $1 \geqslant$ $\left(1+x^{q}\right)^{-1 / q} \geqslant 1-C x$, whenever $x<1 / C$. On the other hand, it is easy to check that if $t>C^{\prime} \varepsilon$, with $C^{\prime}$ large enough, then $a / b<1 / C$. Thus we may write

$$
\int_{C^{\prime} \varepsilon}^{s} a\left(1-C \frac{a}{b}\right) d t \leqslant \int_{C^{\prime} \varepsilon}^{s} \frac{a}{\left((a / b)^{q}+1\right)^{1 / q}} d t \leqslant \int_{C^{\prime} \varepsilon}^{s} a d t .
$$

It is easy to check that

$$
\int_{C^{\prime} \varepsilon}^{s} \frac{a^{2}}{b} d t<C
$$

and that

$$
\left|\int_{C^{\prime} \varepsilon}^{s} a d t-\pi \log \frac{s}{\varepsilon}\right|<C
$$

if $s<t_{0}$. Finally, combining (3.16) with the fact that

$$
\int_{0}^{C^{\prime} \varepsilon} \lambda_{\varepsilon}(t) d t<C
$$


the assertion is proved.

From (3.13), (3.14), and (3.15), we deduce that $\forall r>s>\varepsilon$,

$$
\begin{aligned}
F_{\varepsilon}\left(u, B_{r}\right)-F_{\varepsilon}\left(u, B_{s}\right) & \geqslant \int_{s}^{r} F_{\varepsilon}\left(u, S_{t}\right) d t \geqslant \int_{s}^{r} \lambda_{\varepsilon}\left(\frac{t}{|d|}\right) d t \\
& \geqslant \Lambda_{\varepsilon}\left(\frac{r}{|d|}\right)-\Lambda_{\varepsilon}\left(\frac{s}{|d|}\right) .
\end{aligned}
$$

The lemma is proved.

\subsection{Proof of Proposition 3.1}

First of all, letting $\mathcal{B}\left(s_{0}\right)$ be the family of balls given by Lemma 3.3, we check that properties (2), (3) are verified for $s=s_{0}$. Indeed, from Lemma 3.1,

$$
F_{\varepsilon}\left(u, B_{i} \cap \Omega\right) \geqslant \frac{C_{1} r_{i}}{\varepsilon}
$$

while

$$
\frac{\Lambda_{\varepsilon}(s)}{s} \leqslant \frac{C_{1}}{\varepsilon} .
$$

Thus, the second property is true. Property (3) results directly from the definition of $s_{0}$. Notice that $\{|u|<3 / 4\}$ is contained in the initial family $\mathcal{B}\left(s_{0}\right)$.

Now we let $I$ be the largest interval containing $s_{0}$ such that $\forall s \in I$ there exists a finite family of disjoint balls $\mathcal{B}(s)$ verifying properties (1)(3) above. We already know $I$ is not empty. We now prove that $I$ is open.

Suppose $\left[s_{0}, s_{1}\right] \subset I$. We wish to define a family $\mathcal{B}(t)$ for $t \in\left[s_{1}, s_{1}+\right.$ $\alpha$ ], for some $\alpha>0$. Three cases occur.

Case 1. For the family $\mathcal{B}\left(s_{1}\right)$, all the inequalities in (3) are strict, i.e., $r_{i}\left(s_{1}\right)>s_{1}\left|d_{i}\left(s_{1}\right)\right|, \forall i$ such that $\overline{B_{i}} \subset \Omega$. In this case we let $B_{i}(t)=$ $B_{i}\left(s_{1}\right)$, i.e., we do not change the balls. This defines a family $\mathcal{B}(t)$ of disjoint balls that verifies (1) trivially, (2) also since-by Lemma 3.2$\Lambda_{\varepsilon}(t) / t \leqslant \Lambda_{\varepsilon}\left(s_{1}\right) / s_{1}$ for $t \geqslant s_{1}$. Finally (3) is verified at least when $t \in\left[s_{1}, s_{1}+\alpha\right], \alpha>0$ not too large.

Case 2. There is equality in (3) for, say, balls $B_{1}\left(s_{1}\right), \ldots, B_{\ell}\left(s_{1}\right)$, but all the balls have disjoint closures. In this case the family $\mathcal{B}(t)$ for $t>s_{1}$ is defined by:

- The ball $B_{i}(t), 1 \leqslant i \leqslant \ell$ has the same center as $B_{i}\left(s_{1}\right)$ and its radius $r_{i}(t)$ is such that $r_{i}(t)=t\left|d_{i}(t)\right|=t\left|d_{i}\left(s_{1}\right)\right|$, as long as $\overline{B_{i}(t)} \subset \Omega$, 
which is true when $t \in\left[s_{1}, s_{1}+\alpha\right], \alpha>0$ not too large. (The degree remains constant as $|u|>3 / 4$ on $\mathcal{B}(t) \backslash \mathcal{B}\left(s_{0}\right), \forall t>s_{0}$.) In particular the balls are growing.

- The other balls remain unchanged.

The balls defined this way remain disjoint for $t>s_{1}$ not too large. Property (1) obviously is still true, (3) also. Property (2) remains true in an obvious way for the static balls, as $\Lambda_{\varepsilon}(s) / s$ is decreasing. For the increasing ones, we just use Lemma 3.2, which yields

$$
\begin{aligned}
& F_{\varepsilon}\left(u, B_{i}(t)\right) \\
& \quad \geqslant F_{\varepsilon}\left(u, B_{i}\left(s_{1}\right)\right)+F_{\varepsilon}\left(u, B_{i}(t) \backslash \overline{B_{i}\left(s_{1}\right)}\right) \\
& \quad \geqslant r_{i}\left(s_{1}\right) \frac{\Lambda_{\varepsilon}\left(s_{1}\right)}{s_{1}}+\left|d_{i}(t)\right|\left(\Lambda_{\varepsilon}\left(\frac{r_{i}(t)}{\left|d_{i}(t)\right|}\right)-\Lambda_{\varepsilon}\left(\frac{r_{i}\left(s_{1}\right)}{\left|d_{i}\left(s_{1}\right)\right|}\right)\right) .
\end{aligned}
$$

But, we know that for the growing balls, we have $r_{i}\left(s_{1}\right)=s_{1}\left|d_{i}\left(s_{1}\right)\right|$, and $r_{i}(t)=t\left|d_{i}(t)\right|$. Hence, with the constancy of the degree,

$$
\begin{aligned}
F_{\varepsilon}\left(u, B_{i}(t)\right) & \geqslant r_{i}\left(s_{1}\right) \frac{\Lambda_{\varepsilon}\left(s_{1}\right)}{s_{1}}+\frac{r_{i}(t)}{t} \Lambda_{\varepsilon}(t)-\frac{r_{i}\left(s_{1}\right)}{s_{1}} \Lambda_{\varepsilon}\left(s_{1}\right) \\
& \geqslant r_{i}(t) \frac{\Lambda_{\varepsilon}(t)}{t} .
\end{aligned}
$$

Case 3. There is equality in (3) for some of the balls in $\mathcal{B}\left(s_{1}\right)$ but, say, $\overline{B_{1}\left(s_{1}\right)} \cap \overline{B_{2}\left(s_{1}\right)} \neq \emptyset$. Then we modify the family $\mathcal{B}\left(s_{1}\right)$ as follows: group $B_{1}\left(s_{1}\right)$ and $B_{2}\left(s_{1}\right)$ into a single larger ball $B$ of radius $r_{1}\left(s_{1}\right)+r_{2}\left(s_{1}\right)$. If $B$ intersects, say, $\overline{B_{3}}$, enlarge it so that $B_{1} \cup B_{2} \cup B_{3} \subset B$ and the radius of $B$ is $r=r_{1}+r_{2}+r_{3}$, etc.

Thus, we have a new family of balls $\mathcal{B}^{\prime}\left(s_{1}\right)$ whose closures are disjoint, such that the union of the balls in this family contains the balls of $\mathcal{B}\left(s_{1}\right)$, that verifies properties (2) and (3). This last statement is obviously true for the balls that have not changed in the process. We verify it for a ball $B \in \mathcal{B}^{\prime}\left(s_{1}\right)$ that results from grouping $B_{1}, \ldots, B_{\ell} \in \mathcal{B}\left(s_{1}\right)$.

- Property (2) is verified since

$$
F_{\varepsilon}(u, B) \geqslant \sum_{i=1}^{\ell} F_{\varepsilon}\left(u, B_{i}\right) \geqslant\left(\sum_{i=1}^{\ell} r_{i}\right) \frac{\Lambda_{\varepsilon}\left(s_{1}\right)}{s_{1}}=r \frac{\Lambda_{\varepsilon}\left(s_{1}\right)}{s_{1}} .
$$


- If $\bar{B} \subset \Omega$ and $\operatorname{deg}(u, \partial B)=d \neq 0$ then $d=\sum_{i} d_{i}$, where $d_{i}=$ $\operatorname{deg}\left(u, \partial B_{i}\right)$. Thus

$$
\frac{r}{|d|} \geqslant \frac{r}{\sum_{\left\{i \mid d_{i} \neq 0\right\}}\left|d_{i}\right|} \geqslant s_{1},
$$

since for every $i$ such that $d_{i} \neq 0, r_{i} \geqslant s_{1}\left|d_{i}\right|$. Thus property (3) is verified. If $d=0$, then the property is empty.

Now, to define $\mathcal{B}(t)$ for $t>s_{1}$ we start from $\mathcal{B}^{\prime}\left(s_{1}\right)$, and use case 1 or case 2.

It remains to prove that the interval $I$ is closed. Indeed, suppose $\left[s_{0}, s_{1}\right) \subset I$ and that $\mathcal{B}(s)$ is defined on this interval by the above procedure. We wish to define $\mathcal{B}\left(s_{1}\right)$. First note that since the number of balls in $\mathcal{B}(s)$ is nonincreasing, and since there is initially a finite number of them, the number of balls is constant on some interval $A=$ $\left[s_{1}-\alpha, s_{1}\right)$. On this interval, the balls $B_{1}(s), \ldots, B_{\ell}(s)$ are well defined, have their centers fixed, their radius increases continuously with $s$, and their degree $d_{i}(s)=\operatorname{deg}\left(u, \partial B_{i}(s)\right)$ is constant for $s \in A$. Then we let $B_{i}\left(s_{1}\right)=\bigcup_{s \in A} B_{i}(s)$. This defines a family $\mathcal{B}\left(s_{1}\right)$ of disjoint balls that are easily seen to verify properties (1), (2). For (3), note that $\operatorname{deg}\left(u, \partial B_{i}\left(s_{1}\right)\right)$ is either equal to $d_{i}(s), s \in A$ if $\overline{B_{i}\left(s_{1}\right)} \subset \Omega$ or to 0 . In any case, (3) will be verified.

The proposition is proved. This completes all the proofs.

\section{REFERENCES}

[1] A. Abrikosov, On the magnetic properties of superconductors of the second type, Soviet Phys. JETP 5 (1957) 1174-1182.

[2] L. Almeida and F. Bethuel, Topological methods for the Ginzburg-Landau equations, J. Math. Pures Appl. 77 (1998) 1-49.

[3] F. Bethuel, H. BREZIS and F. HÉlein, Ginzburg-Landau Vortices, Birkhäuser, 1994.

[4] F. BethueL and T. RiviÈre, Vorticité dans les modèles de Ginzburg-Landau pour la supraconductivité, Séminaire E. D. P de l'École Polytechnique XVI (1994).

[5] F. Bethuel and T. RiviÈre, Vortices for a variational problem related to superconductivity, Annales IHP, Analyse non Linéaire 12 (1995) 243-303.

[6] P.G. DeGennes, Superconductivity of Metal and Alloys, Benjamin, New York and Amsterdam, 1966.

[7] R. JERRARD, Lower bounds for generalized Ginzburg-Landau Functionals, SIAM J. Math. Anal. 30 (4) (1999) 721-746.

[8] J. Rubinstein, Six lectures on superconductivity, in: Proc. CRM School on "Boundaries, Interfaces, and Transitions". 
[9] D. Saint-James, G. SARma and E.J. Thomas, Type-II Superconductivity, Pergamon Press, 1969.

[10] E. SANDIER, Lower bounds for the energy of unit vector fields and applications, J. Functional Analysis 152 (2) (1998) 379-403.

[11] E. SANDIER and S. SERFATY, On the energy of type-II superconductors in the mixed phase, Preprint.

[12] S. SERFATY, Solutions stables de l'équation de Ginzburg-Landau en présence de champ magnétique, C. R. A. S. I 326 (8) (1998) 955.

[13] S. SERfATY, Local minimizers for the Ginzburg-Landau energy near critical magnetic field, Part I, Comm. Contemporary Mathematics, to appear.

[14] S. SERFATY, Local minimizers for the Ginzburg-Landau energy near critical magnetic field, Part II, Comm. Contemporary Mathematics, to appear.

[15] S. SERFATY, Stable configurations in superconductivity: Uniqueness, multiplicity and vortex-nucleation, Arch. for Rat. Mech. Anal., to appear.

[16] S. SERFATY, Sur l'équation de Ginzburg-Landau avec champ magnétique, in: Proc. “Journées Équations aux dérivées partielles, Saint-Jean-de-Monts”, 1998.

[17] E.M. STEIn, Harmonic Analysis, Princeton Math. Series, No 43, Princeton University Press, 1969.

[18] D. Tilley and J. Tilley, Superfluidity and Superconductivity, 2nd ed., Adam Hilger, Bristol, 1986. 